SYMBIOSIS

\section{Show me the sugar}

Whereas the virulence factors of pathogenic bacteria are a central focus of many laboratories, the molecular mechanisms that enable symbiotic commensal bacteria to thrive remain largely unexamined. Reporting in Proceedings of the

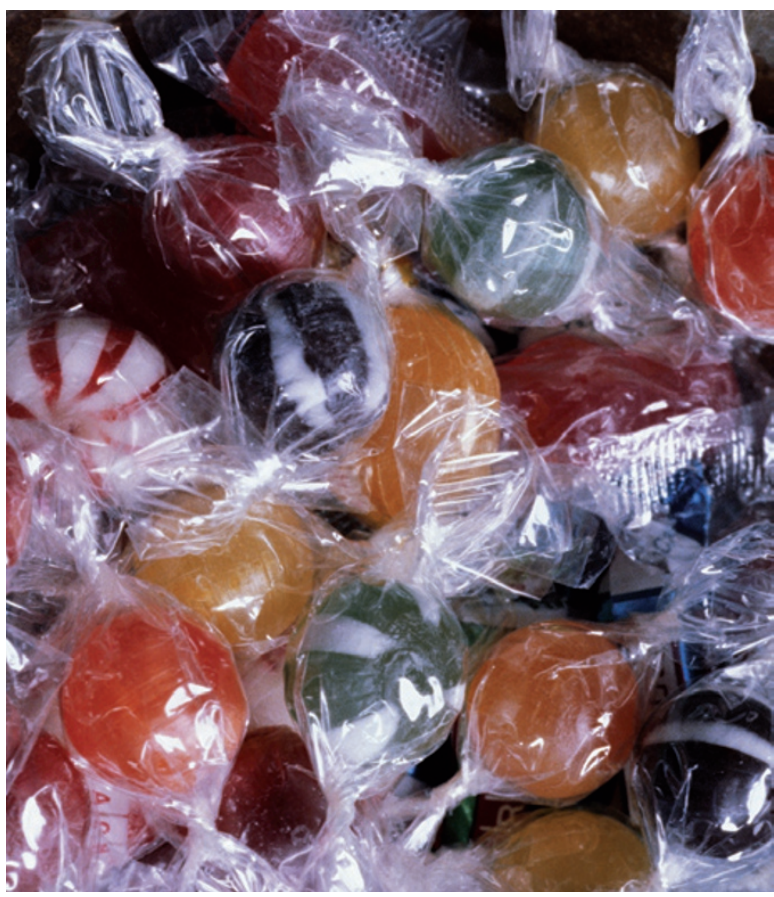

National Academy of Sciences USA, Sarkis Mazmanian and colleagues now show that Bacteroides fragilis needs to be able to regulate surface polysaccharide production to compete during commensal colonization.

Bacteroides spp., which make up a large proportion of the human gut microbiota, encode a number of proteins that synthesize different cell-surface polysaccharides. To determine whether these proteins and their corresponding polysaccharides are important for commensalism, Liu et al. focused on B. fragilis, which encodes at least eight genomic loci (PSA-PSH) that synthesize surface polysaccharides. The expression of seven of these capsular polysaccharides is controlled by the transcriptional regulator multiple promoter invertase (Mpi), and the expression of the remaining polysaccharide (PSC) is controlled independently. By mutating $m p i$ and introducing a deletion into PSC, the authors generated a mutant strain of $B$. fragilis, CPM2, that could not produce any surface polysaccharides. CPM2 exhibited dramatic growth defects in culture, but intriguingly these growth defects reverted after 5 days. Moreover, polysaccharide synthesis was restored in the revertant strain. So, B. fragilis does not tolerate the complete elimination of surface polysaccharides, and finds a way to overcome such detrimental mutations.

To determine the functional importance of these molecules, the authors performed in vivo experiments with germ-free animals. When CPM1, a strain of $B$. fragilis that only expresses PSC, was introduced into germ-free animals, it could efficiently colonize the gut. However, CPM1 was out-competed by wild-type B. fragilis when both strains were introduced together. Furthermore, CPM1 that had been complemented with Mpi, or an Mpi homologue, rapidly out-competed CPM1 alone.

Taken together, these findings indicate that the ability of $B$. fragilis to express certain surface polysaccharides is important for the bacteria to compete effectively during colonization. It will be interesting to determine whether, and how, the rest of the cell envelope contributes to the host-bacteria mutualism in other species.

Asher Mullard

ORIGINAL RESEARCH PAPER Liu, C. H. et al. Regulation of surface architecture by symbiotic bacteria mediates host colonization. Proc. Natl Acad. Sci. USA 105, 3951-3956 (2008) 